gebieten ein Gegenstück hat, nicht von der Hand zu weisen. Es bleibt für unseren Fall die Frage offen, ob eine gleichzeitige Anwendung von Salvarsan und $\mathrm{Hg}$ das Entstehen einer Arzneifestigkeit der Spirochäten verhütet, ob eine summierende Wirkung oder ein hemmender Einfluß auf die Rezeptorentätigkeit zustandekommt. Ferner bedarf die Frage noch einer Klärung, die der Hautklinik von einem Chemiker vorgelegt wurde, ob nicht $\mathrm{Hg}$ und Salvarsan im Körper eine Verbindung eingehen, welche die Wirkung des Salvarsans abschwächt.

Unter den von Ehrlich aufgestellten lehrmäßigen Erwägungen und unter dem Eindruck der ersten klinischen Erfahrungen reiner Salvarsanbehandlung gingen also die meisten Kliniker, bis auf wenige Ausnahmen, zur gleichzeitigen Quecksilber-Salvarsananwendung über und blieben auch hei dieser Behandlungsart, als man durch reichere klinische Erfahrungen und Ausmerzen von Fehlerquellen das Salvarsan viel häufiger und länger im Einzelfall, ja man könnte sagen, ganz anders anzuwenden gelernt hatte als in den ersten Anfängen der Salvarsanzeit. Die Folge war, daß die übergroße Mehrzahl aller Praktiker über die Wirksamkeit reiner Salvarsankuren keine eigenen Erfahrungen sammeln konnte, was angesichts der Bedeutung des Mittels sehr zu bedauern ist. Nur ganz wenige Kliniker waren sich dessen von Anfang an bewußt und deshalb bemüht, reine Salvarsanerfahrungen bei fortschreitendem Ausbau der Salvarsanmethode zu sammeln; unter ihnen ist vor allen Wechselmann zu nennen.

Angesichts seiner Behauptung, mit der reinen Salvarsanbehandlung die gleichen Ergebnisse wie bei der mit $\mathrm{Hg}$ verbundenen erzielen zu können, sah sich auch die Jenaer Hautklinik veranlaßt, Erfahrungen mit jener Kuranordnung zu sammeln. Bevor auf unsere Ergebnisse dieser Behandlungsart eingegangen und diese mit den Erfolgen der gemischten Behandlung verglichen. werden, seien hier einige Arten der Sal.

Aus der Universitäts-Hautklinik in Jena. (Vorstand: Prof. Spiethoff.)

\section{Wirkt die gleichzeitige Anwendung von Salvarsan und Quecksilber summierend?}

\author{
Von Dr. W. Treupel, I. Assistent der Klinik.
}

Schon verhältnismäßig kurze Zeit nach Einführung des Salvarsans, als nach 1-3 Spritzen der erwartete große Erfolg der Therapia magna sterilisans nicht eintrat, ging man zu der mit $\mathrm{Hg}$ verbundenen Salvarsanbehandlung über. Ehrlich selbst sprach dieser Behandlung das Wort, indem er (auf Grund seiner Seitenkettentheorie) von dem Vorhandensein verschiedenartiger Chemorezeptoren ausgehend, auf die Nützlichkeit hinwies, diese, durch therapeutische experimentelle Versuche seiner Schule sichergestellte Tatsache zum Ausgangspunkt einer summierenden Behandlung zu machen. - Es sei aber daran erinnert, daß aus der Ehrlich-Schule auch Versuche vorliegen, die dafür sprechen, da $B$ die gleichzeitige Anwendung verschiedener Stoffe unter Umständen einen hemmenden Einfluß auf die Rezeptorentätigkeit ausüben kann; es kommt eben alles auf die Auswahl der Stoffe an.

Eine weitere Ueberlegung schien Ehrlich für die Salvarsan-Hg-Behandlung zu sprechen, der Gesichtspunkt der Arzneifestigkeit. Ehrlich führt den von Minkowski beobachteten Malariafall an, der zunächst nicht durch Chinin, wohl aber durch Salvarsan sehr gut beeinflußt wurde und der nun bei dem nächsten Anfall auch auf Chinin in gewohnter Weise antwortete. Ehrlich zieht hieraus den Schluß, daß durch Einschaltung eines Stoffes oder gleichzeitige Anwendung zweier Mittel der Arzneifestigkeit vorgebeugt werden kann.

Die Züchtung arzneifester Stämme (Rekurrensspironemen Gonder) ist der Ehrlich-Schule zwar gelungen, Gonder hebt aber ausdrücklich hervor, daß diese mühevoll aufgezwungene Festigkeit der Spironemen gegen Salvarsan in keiner Weise irgendwelche praktische Bedeutung besitzt. Selbst zehn- und zwanzigmalige Einspritzung wird voraussichtlich niemals irgendeine Festigkeit erwirken können.

Scheinen so die Tierversuche nicht sehr dafür zu sprechen, daß man in der Praxis mit dem Entstehen einer Arzneifestigkeit der Spirochäten zu rechnen hat, so sind auf der anderen Seite die alten Erfahrungen über Arzneigewöhnung und die Beobachtung Minkowskis, die auch auf anderen Behandlungsvarsan-Hg-Behandlung angeführt:

E. Hoffman $n$ behandelt neuerdings mit Natrium-Salvarsan und gibt als höchste Einzelgabe bei Männern Dos. 3-4 und bei Frauen Dos. 2-3, wöchentlich einmal in verdünnter Lösung unter gleichzeitiger Hg-Schmierkur.

Gennerich wendet ebenfalls neben Altsalvarsan Quecksilber an, abgesehen von schwangeren oder älteren siechen Frauen und Luesfällen im ersten Zeitpụnkt, bei denen er, nach Entfernung des Primäraffektes, nur Salvarsan anwendet.

K ro m a yer gebraucht Dosen von 1-2 Altsalvarsan, gibt 15 Spritzen im Zeitraume von 4-6 Wochen, in einer Gesamtmenge von $2-3 \mathrm{~g}$ Salvarsan, und läßt eine $\mathrm{Hg}-\mathrm{Kur}$ vorangehen oder folgen.

Scholy gibt zunächst an je zwei aufeinanderfolgenden Tagen je 2 Altsalvarsanspritzen, Dosis 2-3, im ganzen etwa 1 g Altsalvarsan in stark verdünnter Lösung, läßt dann eine Hg-Spritz-Schmierkur von vierwöchiger Dauer folgen, wiederholt hierauf die oben beschriebenen 4 Salvarsanspritzen, nur in etwas milderer Form, und wendet zum SchluB der Kur nochmals eine zwei- bis dreiwöchige Hg-Spritz-Schmierkur an. Bei seiner Kuranordnung steht also die $\mathrm{Hg}$-Behandlung im Vordergrund.

Gegen die Verwendung kleiner Einzelgaben von Dos. 2 und 3 als höchster Satz (nicht als Durchgangsdose), wie sie von E. Hoffmann und Kromayer angewendet werden, muß sich die hiesige Klinik auf Grund ihrer Erfahrungen wenden. Derartige Dosen sind vom reinen Salvarsanstandpunkt aus als ungenügend wirksam zu bezeichnen; mit derartigen Gaben, auch vielen längere Zeit hintereinander gereichten, wurden hier Beobachtungen von Neurorezidiven gemacht, die stark an die erste Salvarsanzeit erinnerten, wo mit ungenügenden Dosen ohne $\mathrm{Hg}$ vorgegangen wurde. E. Hoffmann, Kromayer und Scholz haben diese Folgen nicht erlebt, weil sie entweder gleichzeitig oder vor- oder nachher $\mathrm{Hg}$ anwendeten. Salvarsandosen aber, die bei reiner Salvarsananwendung ungenügend wirken, müssen abgelehnt werden, da das Mittel so ungenügend ausgenützt wird.

Im Gegensatz zu dieser Verbindungsbehandlung steht die reine Salvarsanbehandlung, als deren!Hauptvertreter W echselmann erwähnt wurde.

Wechselmann wendet Alt-, Neu- und Natrium-Salvarsan in wechselnder Form in Einzelgaben von 3-4 intravenös und subkutan an, bis die Wa.R. negativ wird und unter weiteren Spritzen negativ bleibt; über die Gesamtdosen finden sich in seiner Arbeit (B. kl. W.) keine näheren Angaben.

Die Behandlung mit Altsalvarsan hat die hiesige Klinik ganz aufgegeben, da wir bei ihr die noch immer vereinzelt 
gerühmte Ueberlegenheit gegenüber dem Neusalvarsan nicht anerkennen können. - Hier sind mit Neusalvarsan ebenso gute Heilerfolge wie mit Altsalvarsan erzielt; dazu kommt die geringerere Giftigkeit, das damit zusammenhängende seltene Auftreten von Nebenerscheinungen und die einfachere Anwendungsweise der konzentrierten Einspritzung. Die hiesigen Erfahrungen decken sich ganz mit denen von Stern-Düsseldorf. Auch die Anwendung von Natriumsalvarsan hat keinerlei Vorteile gezeigt. Die luetischen Erscheinungen gehen nicht schneller zurück als bei Neusalvarsan, der Wassermann wird nicht schneller negativ, und die Nebenerscheinungen treten etwas häufiger als bei Neusalvarsan auf. Nur eine Berechtigung hat das Natriumsalvarsan: treten nach lange Zeit fortgesetzter Neusalvarsanbehandlung gewisse Empfindlichkeitserscheinungen auf, so läßt sich die Kur, unter Vermeidung der Nebenerscheinungen, durch Uebergang zum Natriumsalvarsan fortsetzen. Dasselbe trifft auch für den umgekehrten Fall zu.

Die verschiedenen Kuranordnungen hiesiger Klinik, die zum Vergleich und zur Beantwortung unserer Frage herangezogen werden sollen, gestalteten sich im einzelnen so:

1. Behandlung mit Neusalvarsan in verdünnter Lösung: Mit Dos. 4 beginnend, gaben wir nach fünf Tagen Dos. 5, von da $a b$ wöchentlich je eine Spritze, indem wir in der Höhe der Einzeldosis bis auf Dosis 8 bzw. 9 stiegen und auf letzterer blieben. - Als Gesamtmenge verabreichten wir 5-6 g Nensalvarsan, immer nach Altsalvarsan berechnet. Als Lösungsmittel wurde destill. $\mathrm{H}_{2} \mathrm{O}$, und zwar auf je $0,15 \mathrm{~g}$ Neusalvarsan $20 \mathrm{ccm}$ gegeben.

2. Dieselbe Behandlung, wie unter 1 beschrieben, aber mit gleichzeitiger Hg-Spritzkur (10 Hg-Spritzen à $0,1 \mathrm{~g} \mathrm{Hg}, \mathrm{Mer}-$ curiolöl).

Folgende Aufstellung gibt eine kurze Uebersicht über die Höhe der bei diesen beiden Kuranordnungen benutzten Salvarsanmengen und der Gaben, die erforderlich waren, um den Wassermann negativ zu gestalten.

Hierzu muß noch bemerkt werden, daß in den Tabellen nur Fälle verzeichnet sind, die hinsichtlich ihrer Kurdurchführung unseren Anforderungen vollkommen entsprochen haben. Die Zahl der Kranken, die, trotz eindringlichster Ermahnungen unserseits, ihre Kur vorzeitig abgebrochen haben, ist leider noch immer sehr groß.

\begin{tabular}{|c|c|c|c|c|}
\hline \multirow{2}{*}{$\begin{array}{c}\text { Salv. in verdünnter } \\
\text { Lösung }\end{array}$} & \multicolumn{2}{|c|}{$\begin{array}{l}\text { Höhe der Dosis bis } \\
\text { Wa.R. - wurde }\end{array}$} & \multicolumn{2}{|c|}{$\begin{array}{c}\text { Höhe der gegebenen } \\
\text { Gesamtdosis }\end{array}$} \\
\hline & ohne $\mathrm{Hg}$ & mit $\mathbf{H g}$ & ohne $\mathrm{Hg}$ & mit $\mathrm{Hg}$ \\
\hline $\begin{array}{l}\text { Primäre Lues (Wa.R.-) : } \\
\text { Primäre Lues (Wa.R. }+ \text { ) : } \\
\text { Sekundäre Lues } \\
\text { Latente Lues (Frühform): } \\
\text { Latente Lues (Spätform): } \\
\text { Tertiäre Lues . . . : }\end{array}$ & $\begin{array}{l}E \\
E \\
\bar{E}\end{array}$ & $\begin{array}{r}. \\
.4,0 \\
4,5 \\
4,6 \\
3,9 \\
5,0\end{array}$ & $\begin{array}{l}3,5 \\
z \\
z\end{array}$ & $\begin{array}{l}\overline{4} \\
4,4 \\
5,1 \\
5,1 \\
4,7 \\
6,1\end{array}$ \\
\hline
\end{tabular}

Aus vorstehender Tabelle ist ersichtlich, daß wir reine Salvarsanbehandlung, Methode 1, damals nur bei primärer Lues mit stets negativem Wassermann und Salvarsan-Quecksilberbehandlung, Methode 2, nur bei primärer Lues mit positivem Wassermann, ferner bei sekundärer und Spätformen der Lues anwandten, und zwar bei Primärfällen in Höhe von $3,5-4,5 \mathrm{~g}$ und bei den übrigen Fällen in Höhe von 4,5-6,0 g. - Die Zahl der beobachteten Fälle und die Länge der jeweiligen Beobachtungsdauer hierbei war kurz folgende:

Primäre Lues. Wa.R. negativ. Reine Salvarsanbehandlung. Zahl der Fälle 7.

Serumreaktion am Schluß der Kur negativ geblieben in 7 Fällen. Hiervon entzogen sich weiterer Beobachtung — Fälle. Hiervon wurden beobachtet 1 Monat 1 Fal

$\left.\begin{array}{rlll}1 & \text { Monat } & 1 & \text { Fall } \\ 2 & \text { Monate } & 1 & , \\ 4 & \prime \prime & 2 & \text { Fălle } \\ 10 & \# & 1 & \text { Fall } \\ 12 & \prime \prime & 1 & ,,\end{array}\right\}$ Wa:R.

Primäre Lues. Wa.R. positiv oder nach der ersten Spritze positiv. $\mathrm{Hg}+$ Salvarsanbehandlung.

Zahl der Fälle 20.

Serumreaktion am Schluß der Kur negativ in 20 Fällen.

Hiervon entzogen sich weiterer Beobachtung 6 Fälle.

Hiervon entzogen sich weiter
Hiervon wurden beobachtet

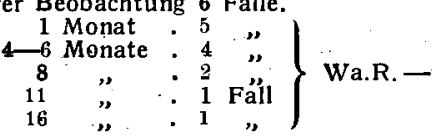

Die Serumreaktion war wieder positiv geworden nach 3 Monaten in 1 Fall.

Sekundäre Lues. $\mathrm{Hg}+$ Salvarsanbehandlung.

Zahl der Fälle 52

Serumreaktion am Schluß der Kur negativ in 52 Fällen.

Hiervon entzogen sich weiterer Beobachtung 14 Fälie.

Hiervon wurden beobachtet 1 Monat . . 13 Fälle

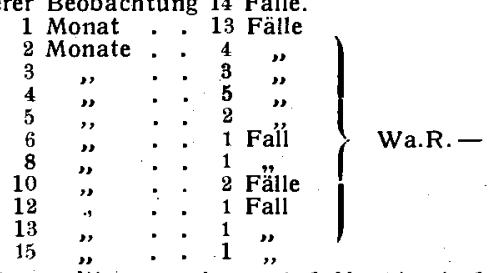

Dle Serumreak:ion war wieder positiv geworden nach 1 Monat in 1 Fall 4 Monaten in 2 Fällen

Latente Lues, Frühform. $\mathrm{Hg}+$ Salvarsanbehandlung.

Zahl der Fälle 13.

Serumreaktion am Schluß der Kur negativ in 10 Fällen.

Hiervon enizogen sich weiterer Beobachtung 4 Fälle.

Hiervon wurden beobachtet .1 Monat . . 1 Fall

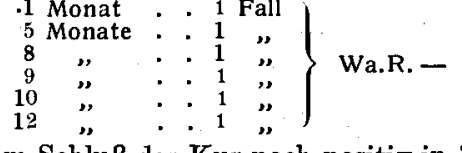

Die Serumreaktion war am Schluß der Kur noch positiv in 3 Fällen. Latente Lues, Spätform. $\mathrm{Hg}+$ Salvarsanbehandlung.

Zahl der Fälle 8.

Serumreaktion am Schluß der Kur negativ in 5 Fällen.

Hiervon entzogen sich weiterer Beobachtung - Fall

Hiervon wurden beobachtet 2 Monate. . . 1 Fall

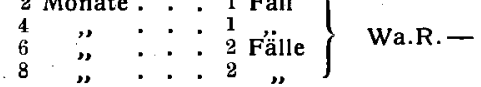

Die Serumreaktion war am Schluß der Kur noch positiv in 3 Fällen. Tertiäre Lues. $\mathrm{Hg}+$ Salvarsanbehandlung.

Zahl der Fälle 15.

Serumreaktion am Schlu B der. Kur negativ in 6 Fällen.

Hiervon entzogen 8ich weiterer Beobachtung 3 Fälle

Hiervon wurden beobachtet ${ }_{9}$ Monate $: 2^{2}$ Fảl $\}$ Wa.R. -

Die Serumreaktion war am Schluß der Kur noch positiv in 9 Fällen. maßen:

Die nächste Kuranordnung 3 gestaltete sich folgender-

Mit Dos. 1 Neusalvarsan beginnend, verabreichten wir nach fünf Tagen als zweite Einspritzung Dos. 2 Neusalvarsan und stiegen in 8tägigen Abständen bis Dos. 4 Neusalvarsan bei Frauen und Dos. 5 Neusalvarsan bei Männern, um dann auf dieser jeweils erreichten Einzelgabe zu bleiben und diese solange weiter zu geben, bis. Wassermann und Stern negativ geworden und im Verlauf von sechs weiteren Einspritzungen beständig negativ geblieben waren. Als Lösungsmittel verwendeten wir auf je $0,1 \mathrm{~g}$ Neusalvarsan $1 \mathrm{ccm}$ frisch destilliertes Wasser. Eine bestimmte Gesamtgabe wurde hier nicht festgesetzt, über die Höhe der jeweils gegebenen Gesamtmenge unterrichtet die nächste Aufstellung Nr. 2.

Gewöhnlich behandelten wir 3-4 Monate lang; war der gewünschte Heilerfolg noch nicht eingetreten, so wurde nach einer Pause von etwa 6-8 Wochen eine Nachkur, ähnlich der 1. Kur, angeschlossen.

Die Kuranordnung 4 ist die gleiche wie 3, nur gaben wir bei ihr gleichzeitig eine Hg-Spritzkur wie bei der 1. Anordnung.

Im allgemeinen beobachten wir unsere Kurerfolge in der Weise, daß wir zu Beginn der Kur Blut entnehmen; falls hierbei z. B. bei Lues 1 die Wa.R. negativ war, so wird nach der ersten Einspritzung nochmals Blut genommen, wobei man oft ein Umschlagen der vorher negativen Reaktion beobachtet. Während der eigentlichen Kur stellen wir dann die Wa.R. nach der 4., 6., 8. etc. Einspritzung an. Bezüglich der Wa.R. verstehen wir unter Negativwerden und -bleiben dieser Reaktion nicht nur ein Negativwerden der Original-Wa.R., sondern auch der Sternschen Modifikation, die bekanntlich meist viel später negativ wird als die Originalmethode. Auch auf zweifelhafte Stern-Reaktionen soll man bei der Durchführung der Kur achten, da sie oft genug Vorboten späteren Positivwerdens auch der Originalmethode sind. Wie an anderer Stelle, wird auch hier auf die Verwendung von zwei Extrakten Wert gelegt. Wohl wegen der Hinzunahme der Sternschen Modifikation haben wir nicht so hohe Zahlen von Heilung zu verzeichnen, wie z. B. 
Gennerich, der stets nur von Wa.R. und nicht von der Sternschen Modifikation spricht.

Zur Nachprüfung werden in Abständen von vier Wochen nach beendeter Kur Blutentnahmen gemacht, nach einem Vierteljahr die erste, nach je zwei weiteren Halbjahren die zweite und dritte Probespritze nach den Angaben von Gennerich. Eine Wiederholung der Kur wird nur vorgenommen, wenn sie durch ein etwaiges Neuaufflackern der Erkrankung in klinischer oder serologischer Hinsicht bedingt ist. - Hierbei sei besonders auch noch der Untersuchung der Zerebrospinalflüssigkeit gedacht, die berufen ist, neben der Wa.R. eine große Rolle $\mathrm{zu}$ spielen.

\begin{tabular}{|c|c|c|c|c|}
\hline \multirow{2}{*}{ Salv. in konz. Lösung } & \multicolumn{2}{|c|}{$\begin{array}{l}\text { Höhe der Dosis bis } \\
\text { Wa.R. - wurde }\end{array}$} & \multicolumn{2}{|c|}{$\begin{array}{l}\text { Höhe der gegebenen } \\
\text { Gesamtdosis }\end{array}$} \\
\hline & ohne $\mathrm{Hg}$ & mit $\mathrm{Hg}$ & ohne $\mathrm{Hg}$ & mit $\mathrm{Hg}$ \\
\hline 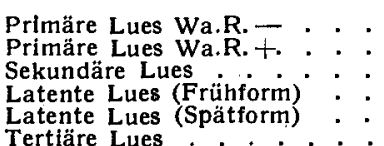 & $\begin{array}{l}2,6 \\
3,3 \\
3,8 \\
2,0 \\
4,7 \\
3,4\end{array}$ & $\begin{array}{l}3,2 \\
3,3 \\
3,4 \\
4,7 \\
3,8 \\
2,9\end{array}$ & $\begin{array}{l}2,6 \\
4,7 \\
5,5 \\
4,0 \\
6,3 \\
4,9\end{array}$ & $\begin{array}{l}3,2 \\
4,5 \\
45 \\
5,9 \\
5.6 \\
4,6\end{array}$ \\
\hline
\end{tabular}

Aus der vorstehenden Uebersicht ergibt sich, daß hinsichtlich des Negativwerdens der Wa.R. bei der gemischten gegenüber der reinen Salvarsanbehandlung keinerlei Vorteile zu verzeichnen sind. Die erforderliche Salvarsanmenge, bei der die Wa.R. negativ wird und bleibt, ist bei reiner Salvarsanbehandlung eher niedriger als bei der mit $\mathrm{Hg}$ verbundenen. - Die Zahl der beobachteten Fälle und die Lännge der jeweiligen $\mathrm{Be}$ obachtungsdauer hierbei war folgende:

Primäre Lues. Wa.R. negativ. Reine Salvarsanbehandlung.

Zahl der Fälle 6.

Serumreaktion am Schlu $\beta$ der Kur negativ geblieben in 6 Fällen Hiervon entzogen sich weiterer Beobachtung 2 Fälle.

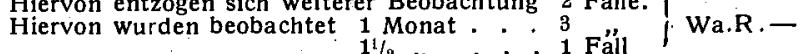

Primäre Lues. Wa.R. negativ. Hg + Salvarsanbehandlung.

Zahl der Fälle 3.

Serumreaktion am Schlu $\beta$ der Kur negativ geblieben in 3 Fällen. Hiervon entzogen sich weiterer Beobach tung 1 Fall.

Hiervon wurden beobachtet 3 Monate : : 1 " 16 Wa.R. -

Primäre Lues. Wa.R. positiv oder nach der ersten Spritze positiv. Reine Salvarsanbehandlung.

Zahl der Fälle 19.

Serumreaktion am Schluß der Kur negativ in 19 Fällèn.

Hiervon entzogen sich weiterer Beobachtung 8 Fälle.

Hiervon wurden beobachtet 1 Monat . 1 Fall

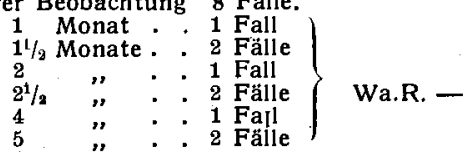
Die Serumreaktion war wieder positiv geworden nach $\frac{1}{1 / 2 \text { Monat in } 1 \text { Fall }}$

Primäre Lues. Wa.R. positiv oder nach der ersten Spritze positiv. $\mathrm{Hg}+$ Salvarsan behandlung.

Zahl der Fälle 13.

Serumreaktion am Schluß der Kur negativ in 13 Fällen.

Hiervon entzogen sich welterer Beobachtung 2 Fälle.

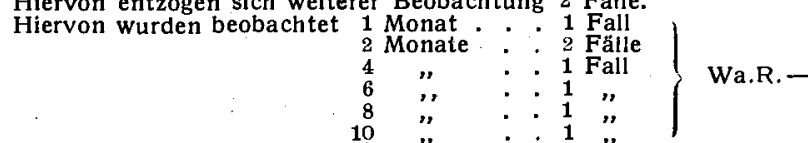

Die Serumreaktion war wieder positiv geworden nach $1 / 2$ Monat in 1 Fall 7 Monaten in 1 ,"

Sekundäre Lues. Reine Salvarsanbehandlung.

Zahl der Fälle 32 .

Serumreaktion am Schluß der Kur negativ in 32 Fällen.

Hiervon entzogen sich weiterer Beobachung 9 Fälle.

Hiervon wurden beobachtet 1 Monat. ${ }_{1}^{1 / 2}$ Monate $: 1$ Fäl

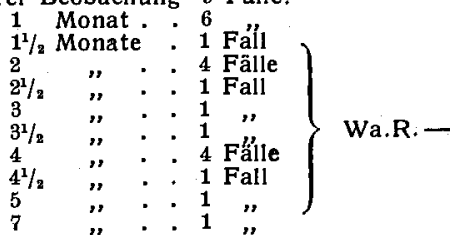

Die Serumreaktion war wieder positlv geworden nach 2 Monaten in 2 Fällen. Sekundäre Lues. $\mathrm{Hg}+$ Salvarsanbehandlung.

Zahl der Fälle 31.

Serumreaktion am Schluß der Kur negativ in 31 Fällen.
Hiervon entzogen sich weiterer Beobachtung 13 Fälle. Hiervon wurden beobachtet 1 Monat. . . 3 Fall

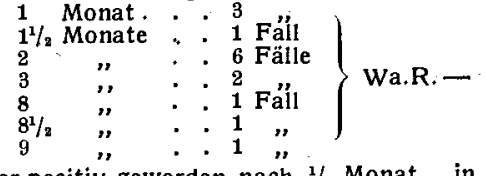

Die Serumreaktion war wieder positiv geworden nach $1 / 2$ Monat in 1 Fal 2 Monaten in 1 ,",

Latente Lues, Frühform. Reine Salvarsanbehandlung.

Zahl der Fälle 2.

Serumreaktion am Schluß der Kur negativ in 2 Fällen. Hiervon entzogen sich welterer Beobachtung 1 Fall
Hiervon wurden beobachtet 1 Monat Wa.R. Latente Lues, Frühform. Hg + Salvarsanbehandlung. Zahl der Fälle 3.

Serumreaktion am Schluß der Kur negativ in 3 Fällen.

Hiervon entzogen sich weiterer Beobachtung 1 Fall Hiervon wurden beobachtet 3 Monate. . . 1 1 " $\}$ Wa.R. -

Latente Lues, Spätform. Reine Salvarsanbehandlung. Zahl der Fälle 9 .

Serumreaktion am Schluß der Kur negativ in 8 Fällen. Hiervon entzogen sich welterer Beobachtung 5 Fälle $\left.\begin{array}{r}\text { Hiervon wurden beobachtet } 1 \text { Monat } 2 \text { Monate } 1 \text { Fall } \\ 3\end{array}\right\}$ Wa.R. Die Serumreaktion war am Schluß der Kur noch positiv in 1 Falle. Latente Lues, Spätform. Hg + Salvarsanbehandlung.

Zahl der Fälle 6.

Serumreaktion am Schluß der Kur negativ in 4 Fällen. Hiervon entzogen sich welterer Beobachtung - Fälle Hiervon wurden beobachtet 3 Monate : : : ${ }_{3}^{1 / 2}, 1$ Fäll

Die Serumreaktion war am Schluß der Kur noch positiv in 2 Fällen. Tertiäre Lues. Reine Salvarsanbehandlung.

Zahl der Fälle 5.

Serumreaktion am Schluß der Kur negativ in 4 Fällen. Hiervon entzogen sich weiterer Beobachtung 2 Fäle Hiervon wurden beobachtet 1 Monat. . . 1 Fall, der stets negatlv war 2 Monate . . 1 Fall. und blieb

Die Serumreaktion war am Schluß der Kur noch positiv in 1 Falle.

Tertiäre Lues. $\mathrm{Hg}+$ Salvarsanbehandlung.

Zahl der Fälle 16.

Serumreaktion am Schluß der Kur negativ in 5 Fällen.

Hiervon entzogen sich weiterer Beobachtung 1 Fall

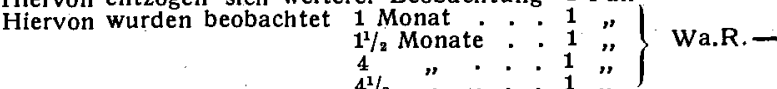

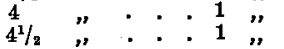

Die Serumreaktion war am Schluß der Kur noch positiv in 11 Fällen.

Der Kurerfolg ist nach unseren Erfahrungen in beiden Fällen der gleiche, wenn nicht bei der reinen Salvarsanbehandlung noch um ein geringes günstiger. Auch die Dauerheilungen sind bei der gemischten Art nicht besser.

Die eingangs aufgestellte Frage: wird bei gleichzeitiger Anwendung von Salvarsan und $\mathrm{Hg}$ Arzneifestigkeit vermieden? ist an der Hand unserer. Beobachtungen dahin zu beantworten, daß sich Anhaltspunkte für das Ents teh en einer Arzneifestigkeit unter der Kur nicht sicher entdecken lassen. Die zweite Frage: findet bei gleichzeitiger Anwendung der beiden Mittel eine summierende Wirkung statt? muß auf Grund hiesiger Erfahrungen verneint werden.

Diese Feststellungen brauchen nicht den Ausschluß von $\mathrm{Hg}$ zu bedeuten. Es liegen hier genügend Beobachtungen vor, die den Nutzen des $\mathrm{Hg}$ neben Salvarsan in manchen Fällen dartun, wenn $\mathrm{Hg} \mathrm{nach}$ einer vollen Salvarsankur angewendet wird. Auf Grund solcher Beobachtungen werden jetzt Erfahrungen mit folgender Kuranordnung gesammelt:

Anwendung reiner Salvarsanbehandlung, bis die Wa.R. negativ wird und unter der weiteren Kur negativ bleibt, oder aber bis zu dem Augenblick, wo der Eindruck besteht, daß sich die Wirksamkeit des Salvarsans in dem betreffenden Fall erschöpft hat; in Gramm Salvarsan ausgedrückt, nicht unter $4 \mathrm{~g}$ und durchschnittlich nicht über 6 g Salvarsan, dann eine vollwertige Hg-Mercinol-Kur zehn Wochen hindurch, wöchentlich einmal $0,1 \mathrm{~g} \mathrm{Hg}$, daran anschließend wieder Salvarsan, mindestens $3 \mathrm{~g}$, unter Umständen noch wesentlich mehr. Die Höhe der Einzelgaben und der Zeitabstand zwischen den einzelnen Spritzen bleibt derselbe wie bei der einleitenden Kur. - Im ersten Abschnitt der Lues bei negativer und auch im Verlauf 
der Kur negativ bleibender Wa.R. wird hier nach wie vor allein Neusalvarsan angewendet, in einer Gesamthöhe von $2,5-3 \mathrm{~g}$ (immer berechnet nach Altsalvarsan). Ist die Wa.R. schon zu diesem Zeitpunkt positiv, so verabfolgen wir eine volle Kur, wie bei Lues 2, ist die Wa.R. zu Beginn der Kur nur angedeutet positiv und wird sie nach den ersten Einspritzungen ganz negativ, oder tritt erst nach der ersten Spritze eine positive Reaktion ein, aber nur vorübergehend, so geben wir eine abgeschwächte Vollkur.

Professor Spieth off vertritt den Standpunkt, nicht grundsätzlich intermittierend $\mathrm{zu}$ behandeln, sondern durch eine möglichst lange, fortlaufende Kur zu erreichen, was zu erreichen ist, unter voller Ausnutzung des Salvarsans und strenger Anlehnung an den Wegweiser der Wa.R.

Zusammenfassung. 1. Eine summierende Wirkung bei gleichzeitiger Salvarsan-Hg-Behandlung findet nicht statt.

2. Das bedeutet zunächst noch nicht den Verzicht auf $\mathrm{Hg}$ bei der Durchschnittsbehandlung, sondern weist nur auf eine andere Kurorandnung hin.

3. Der sicherste Weg zur Vermeidung schwerer Nebenwirkungen des Salvarsans ist, abgesehen von der strengen Beobachtung schon feststehender Gegenanzeigen, die Verwendung kleiner Anfangsgaben und die allmähliche Steigerung der Gaben bei Frauen bis Dosis 4 , bei Männern bis Dosis 5 .

4. Durchschnittsgaben bis zu Dosis 3 sind als ungenügend wirksame Salvarsanmengen und daher, allein verabreicht, als nicht unbedenklich zu bezeichnen.

Literatur: P. Ehrlich, Aus Theorie und Praxis der Chemotherapie. Gennerich, Hamburg. Med. Ueberseehefte 1914 Nr. 2. - Wechselmann, B. kl. W. 1914 Nr. 12. S c h o lz, B. kl. W. 1914 Nr. 33. 二 Kroma yer, D: Ueber den Stand der Luestherapie. Vortrag in der Sektion $f$. Heilkunde d. med.naturw. Ges. in Jena (10. Juli 1913). 